\title{
General treatment for dark energy thermodynamics
}

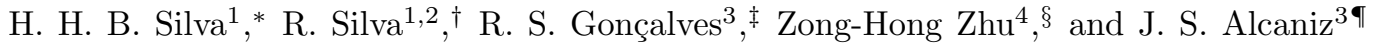 \\ ${ }^{1}$ Universidade Federal do Rio Grande do Norte, Departamento de Física, Natal - RN, 59072-970, Brasil \\ ${ }^{2}$ Universidade do Estado do Rio Grande do Norte, \\ Departamento de Física, Mossoró-RN 59610-210, Brasil \\ ${ }^{3}$ Observatório Nacional, 20921-400 Rio de Janeiro - RJ, Brasil and \\ ${ }^{4}$ Department of Astronomy, Beijing Normal University, Beijing 100875, China
}

(Dated: April 19, 2022)

\begin{abstract}
In this work we discuss a general approach for the dark energy thermodynamics considering a varying equation of state (EoS) parameter of the type $\omega(a)=\omega_{0}+F(a)$ and taking into account the role of a non-zero chemical potential $\mu$. We derive generalized expressions for the entropy density, chemical potential and dark energy temperature $T$ and use the positiveness of the entropy to impose thermodynamic bounds on the EoS parameter $\omega(a)$. In particular, we find that a phantom-like behavior $\omega(a)<-1$ is allowed only when the chemical potential is a negative quantity $(\mu<0)$.
\end{abstract}

PACS numbers: 98.80.-k, 95.36.+x, 95.30.Tg

\section{INTRODUCTION}

The discovery of the cosmic acceleration is certainly one of the most important cosmological results of the last decades. It implies that either the Universe is currently dominated by an exotic dark energy component or that the Einstein's general theory of relativity breaks down at very large scales [1. The profound implications of this discovery have motivated observational efforts to understand the mechanism behind the cosmic acceleration, with a number of experiments that aim to map the history of expansion and growth of structure with percent-level precision being planned for the next years. From the physical point of view, several fundamental questions on the origin and nature of the dark energy still remain completely opened. Certainly among these, the thermodynamical behavior of this component plays an important role not only in constraining its time evolution 22 but also in predicting the dynamical and thermodynamical fate of the Universe 3 .

The thermodynamic behavior of the dark energy considering a constant equation-of-state parameter (EoS) $\omega$ with null chemical potential $(\mu=0)$ was discussed in [3. The authors found that not only a phantom behavior $(\omega<-1)$ is forbidden by the second law of thermodynamics but also that the temperature associated to this component increases with the cosmic expansion (see also 44). Later on, several authors [5, 6] extended these results considering a non-zero chemical potential $\mu$. They showed that for values of $\mu<0$, the EoS parameter may cross the so-called phantom divide line from which the fluid acquires a phantom-like behavior. More recently, we studied some thermodynamic aspects of a

\footnotetext{
* heydson@dfte.ufrn.br

$\dagger$ raimundosilva@dfte.ufrn.br

$\ddagger$ rsousa@on.br

$\S$ zhuzh@bnu.edu.cn

alcaniz@on.br
}

dark energy component described by a varying EoS parameter [2]. We found that the time-dependent term behaves like a bulk viscosity in the temperature evolution law and placed some thermodynamic bounds on the parameters $\omega_{0}$ and $\omega_{a}$ from the second law of thermodynamics and from the positiveness of the entropy - where we have assumed the statistical microscopic concept of entropy, i.e. $S=k_{B} \ln W>0$.

In this paper, we develop a general treatment for the dark energy thermodynamics considering (i) non-zero chemical potential and (ii) a time-dependent EoS parameter. Without loss of generality, we parameterize the function $\omega(a)$ as

$$
\omega(a) \equiv \frac{p_{x}}{\rho_{x}}=w_{0}+F(a)
$$

with encompasses most of the relevant cases discussed in the literature, e.g., $F(a)=\omega_{a}(1-a)$ 7, 8, $F(a)=-\omega_{a} \ln a \quad[9] ; \quad F(a)=\omega_{a}(1-a) /\left(1-2 a+2 a^{2}\right)$ [10] (see also 11 for other parameterizations).

From this background, new evolution laws of some physical quantities such as entropy density and chemical potential are obtained and new constraints are imposed to $\omega(a)$ through the positiveness of the entropy. Therefore, considering this thermodynamic condition, we also find that, although values $\mu<0$ are compatible with phantom models without the need to consider negative temperatures (as argued by [5, 6]), for $\mu \geq 0$ phantom cosmology becomes physically meaningless. We also show that most of the results discussed earlier in the literature are particular cases of the treatment discussed here. Throughout this paper a subscript 0 stands for present-day quantities and a dot denotes time derivative. We use the speed of light as $c=1$.

\section{THERMODYNAMICS OF DARK ENERGY}

Let us consider a flat, homogeneous and isotropic cosmological model described by the Friedmann-Robertson- 
Walker (FRW) metric:

$$
d s^{2}=d t^{2}-a^{2}(t)\left(d r^{2}+r^{2} d \theta^{2}+r^{2} \sin ^{2} \theta d \phi^{2}\right),
$$

In this scenario, the dark energy component is considered as a relativistic perfect fluid, in which its equilibrium thermodynamic states are described by an energy momentum tensor

$$
T^{\delta \nu}=\rho_{x} u^{\delta} u^{\nu}-p_{x} h^{\delta \nu},
$$

a particle current,

$$
N^{\delta}=n u^{\delta},
$$

and an entropy current,

$$
S^{\delta}=s u^{\delta}=n \sigma u^{\delta},
$$

where $h^{\delta \nu} \equiv g^{\delta \nu}-u^{\delta} u^{\nu}$ is the usual projection tensor; $n$ is the particle number density; $s$ and $\sigma$ are the entropy density and the specific entropy (per particle) respectively [12]. The conservation of $T^{\delta \nu}$ and $N^{\delta}$ provides the following relations

$$
\begin{gathered}
u_{\nu} T^{\delta \nu} ; \delta=\dot{\rho}_{x}+3\left(\rho_{x}+p_{x}\right) \frac{\dot{a}}{a}=0, \\
N^{\delta} ; \delta=\dot{n}+3 n \frac{\dot{a}}{a}=0,
\end{gathered}
$$

where semi-colons mean covariant derivative. Integrating the above expressions, it is straightforward to show for a general $\omega(a)$ that

$$
\begin{gathered}
\rho_{x}=\rho_{0}\left(\frac{a}{a_{0}}\right)^{-3} \exp \left[-3 \int \frac{\omega(a)}{a} d a\right], \\
n=n_{0}\left(\frac{a}{a_{0}}\right)^{-3} .
\end{gathered}
$$

In order to derive the evolution law of dark energy temperature, first we consider that the physical quantities $p_{x}, \rho_{x}, n$ and $\sigma$ are related to the temperature $T$ trough the Gibbs law: $n T d \sigma=d \rho_{x}-\frac{\rho_{x}+p_{x}}{n} d n$. Assuming that $\rho_{x}=\rho_{x}(n, T)$ and $p_{x}=p_{x}(n, T)$, it can be shown that such evolution is given by [13 15]

$$
\frac{\dot{T}}{T}=\left(\frac{\partial p_{0}}{\partial \rho_{x}}\right)_{n} \frac{\dot{n}}{n}+\left(\frac{\partial \Pi}{\partial \rho_{x}}\right)_{n} \frac{\dot{n}}{n},
$$

where the dark energy pressure is composed of a constant term $\left(p_{0}\right)$ and by another time-dependent term ( $\left.\Pi\right)$ as follow

$$
p_{x} \equiv p_{0}+\Pi=\omega_{0} \rho_{x}+F(a) \rho_{x}=\omega(a) \rho_{x} .
$$

The above equations provide the relation

$$
T=T_{0} \exp \left[-3 \int \frac{\omega(a)}{a} d a\right] .
$$

It should be noted that the dark energy temperature is always positive and growing in the course of the universe expansion regardless of the $\omega(a)$ value (because the EoS parameter is a negative quantity). A likely physical meaning for this behavior is that thermodynamic work is being done on the system through an adiabatic expansion [3] (For a alternative thermodynamics approach which the fluid takes temperature negative values see, e.g., 4, 16]).

On the other hand, Eq. (6) can be rewritten as

$$
\dot{\rho}_{x}+3\left(\rho_{x}+p_{0}\right) \frac{\dot{a}}{a}=-3 \Pi \frac{\dot{a}}{a} .
$$

This means that the varying part of the dark energy pressure $\Pi$ plays the role of an entropy source term and, therefore, the $\omega(a)$-fluid description assumed here mimics a fluid with bulk viscosity. It suggests a more complete form of entropy density evolution which is different from the widely used expression [3, 5, 6]. Our starting point is the well-known Euler relation: $T s=\rho_{x}+p_{x}-\mu n$. From equations (8), 12) and the comoving volume $V \propto a^{3}$, we find

$$
\sigma \equiv \frac{S}{N}=\frac{\rho_{0}}{T_{0}} \frac{V_{0}}{N}[1+\omega(a)]-\frac{\mu_{0}}{T_{0}},
$$

where we used the condition $\mu / T=\mu_{0} / T_{0}$ which will be demonstrated below using the definition of Gibbs free energy. Therefore, using the above relation and taking the covariant derivative of Eq. (5) we obtain

$$
S^{\alpha}{ }_{\alpha}=\dot{s}+3 s \frac{\dot{a}}{a}=\frac{\rho_{0}}{T_{0}} \frac{V_{0}}{V} \dot{\omega}(a),
$$

or still

$$
\frac{d s}{d a}+\frac{3 s}{a}=\frac{\rho_{0}}{T_{0}} \frac{V_{0}}{V} \omega^{\prime}(a) .
$$

whose solution is

$$
s=a^{-3}\left(s_{0}+\frac{\rho_{0}}{T_{0}}\left[\omega(a)-\omega_{0}\right]\right) .
$$

Note that for a constant EoS parameter $\left(\omega(a) \rightarrow \omega_{0}\right)$ we recovered the entropy relation derived in Refs. [3, 5, 6].

Another relevant physical quantity in thermodynamics analysis is the chemical potential $\mu$. A way to include it in our treatment is using the Gibbs free energy [17]

$$
G(T, p, N) \equiv U+p V-T S
$$

with

$$
\mu=\left(\frac{\partial G}{\partial N}\right)_{T, p} .
$$

Thus, using the equations (8), (9), 12) and (17) we find that

$$
G=N\left[\frac{\rho_{0}}{n_{0}}\left(1+\omega_{0}\right)-\frac{T_{0} s_{0}}{n_{0}}\right] \frac{T}{T_{0}},
$$




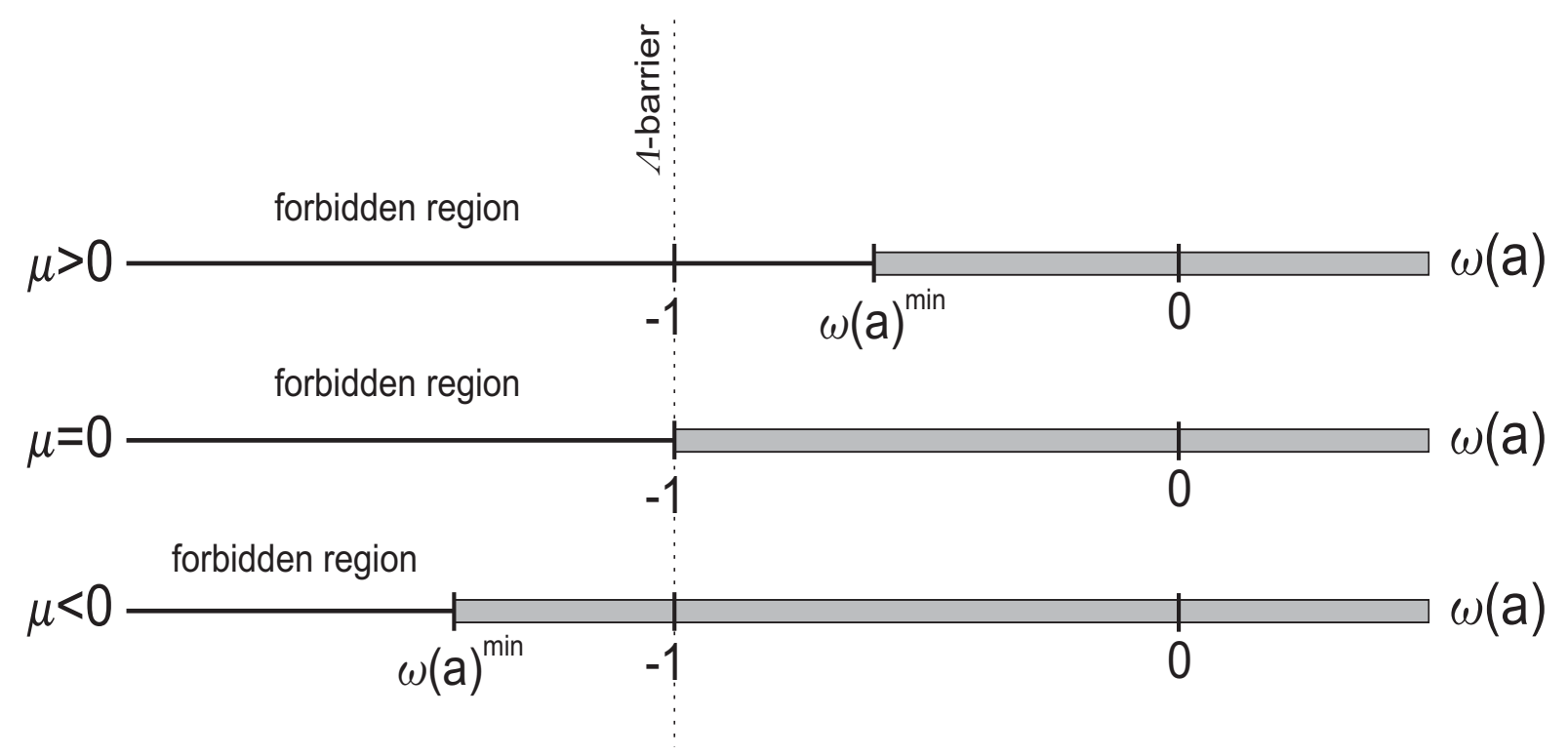

FIG. 1. Representation of thermodynamic constraint [Eq. 24)] for the cases $\mu<0, \mu=0$ and $\mu>0$. The light gray bars represent the values allowed for the parameters in order to satisfy the positiveness of the entropy. The black lines represent the values that do not obey this latter condition (forbidden region) whereas the dashed line represents the cosmological constant limit. Note that for $\mu<0$ the phantom-like regime is allowed, but for $\mu=0$ and $\mu>0$, it is forbidden.

and consequently

$$
\mu=\mu_{0}\left(\frac{T}{T_{0}}\right)=\mu_{0} \exp \left[-3 \int \frac{\omega(a)}{a} d a\right]
$$

where

$$
\mu_{0} \equiv\left[\frac{\rho_{0}}{n_{0}}\left(1+\omega_{0}\right)-\frac{T_{0} s_{0}}{n_{0}}\right]
$$

is the present-day value of the chemical potential. Note that the temperature and chemical potential evolve in the same way (less than a constant) regardless if the dark energy EoS is constant or time-dependent. This is so because for both treatment the condition $\mu / T=\mu_{0} / T_{0}$ is always kept [5, 6, 18].

\section{THERMODYNAMIC CONSTRAINTS}

The thermodynamics formalism developed in the previous section leads us to ask the following questions: which constraints can be imposed to the EoS parameter and the chemical potential in order to satisfy the positiveness of the entropy? Is the phantom-like behavior of the dark energy fluid allowed? If so, for which parameter interval?

Taking the above relations we write the total entropy as

$$
S=\left(\frac{\rho_{0}[1+\omega(a)]-\mu_{0} n_{0}}{T_{0}}\right) V_{0},
$$

whose positivity requires that

$$
\omega(a) \geq \omega(a)^{\text {min }}=-1+\frac{\mu_{0} n_{0}}{\rho_{0}} .
$$

Note that in the limit $\omega(a)^{\text {min }} \rightarrow \omega_{0}^{\text {min }}$ we recover the results for constant EoS [5, 6]. Furthermore, we have a limit for the time-dependent EoS parameter, below which the entropy becomes negative (see, e.g, [16, 18, 20] for other thermodynamic analyses).

The minimal value $\omega(a)^{\text {min }}$ depends explicitly on the chemical potential. Therefore, firstly we consider $\mu=$ $\mu_{0}=0$. In this case, we have $\omega(a)^{\min }=-1$ and a phantom-like behavior of the dark energy fluid is forbidden. On the other hand, for $\mu>0$, the minimal value does not reach the cosmological constant point $\omega(a)=\omega_{0}=-1$ and again a phantom-like regime is forbidden. Finally, if the chemical potential is negative, values of $\omega(a)<-1$ are allowed. A graphical representation of these bounds is shown in Fig. 1. In particular, in the limit $\omega(a) \rightarrow \omega_{0}$, the results obtained in Ref. [5, 6] are readily recovered.

In Table I we present the constraints obtained through positivity of entropy. Note that most of the results presented in the literature are particular cases of Eqs. (24). 
TABLE I. Thermodynamic constraints on $\omega(a)$. All particular cases are shown for the different values of the temperature $(T)$, the chemical potential $(\mu)$ and of the function $F(a)$.

\begin{tabular}{|c|c|c|c|c|}
\hline Reference & $T$ & $\mu$ & $F(a)$ & $\omega(a)^{\min }$ \\
\hline This work & $>0$ & $\neq 0$ & $\neq 0$ & $-1+\frac{\mu_{0} n_{0}}{\rho_{0}}$ \\
\hline 2 & $>0$ & $=0$ & $\neq 0$ & -1 \\
\hline 18 & $<0$ & $\neq 0$ & $\neq 0$ & - \\
\hline 16 & $<0$ & $=0$ & $=0$ & - \\
\hline 5. 6. & $>0$ & $\neq 0$ & $=0$ & $-1+\frac{\mu_{0} n_{0}}{\rho_{0}}$ \\
\hline 4 & $<0$ & $=0$ & $=0$ & - \\
\hline 3 & $>0$ & $=0$ & $=0$ & -1 \\
\hline
\end{tabular}

For instance, for $\mu=\mu_{0}=0$ we recovered the results of [2] whereas if one assumes $\omega(a) \rightarrow \omega_{0}$ we obtain the results of [5, 6; Finally, considering $\mu=\mu_{0}=0$ and $\omega(a) \rightarrow \omega_{0}$ we recovered the results of [3]. Note also that the results obtained by [4, 16, 18] assuming $T<0$, can also be mapped in the present investigation.

\section{CONCLUSIONS}

We have discussed a general treatment for the dark energy thermodynamics by considering a varying EoS parameter and non-zero chemical potential. We have derived general equations for the dark energy entropy and chemical potential and imposed thermodynamic constraints for the $\omega$-parameter in order to satisfy the positiveness of the entropy. These bounds are displayed in Figure 1, where it is clear that, as the minimal value $\omega(a)^{\text {min }}$ depends explicitly upon the chemical potential, only the case $\mu<0$ allows the phantom-like behavior without considering negative temperature (as discussed by [4, 18]). The treatment discussed here generalizes most of the results investigated in the literature, as summarized in Table I. Finally, we emphasize that this procedure can be extended to include non-minimal coupling between dark matter and dark energy. This issue will be addressed in a forthcoming communication.

\section{ACKNOWLEDGMENTS}

H.H.B.S., R.S., R.S.G. and J.S.A. are very grateful to FAPERJ, CNPq and CAPES for the grants under which this work was carried out. Z.-H.Z. is supported by Chinese Ministry of Science and Technology National Basic Science Program (Project 973) under Grant No. 2012CB821804 and National Natural Science Foundation of China under Grants Nos. 11073005 and 11373014.
[1] V. Sahni and A. A. Starobinsky, Int. J. Mod. Phys. D 9, 373 (2000); P. J. E. Peebles and B. Ratra, Rev. Mod. Phys. 75, 559 (2003); T. Padmanabhan, Phys. Rep. 380, 235 (2003); J. S. Alcaniz, Braz. J. Phys. 36, 1109 (2006); R. R. Caldwell and M. Kamionkowski, Ann. Rev. Nucl. Part. Sci. 59, 397 (2009); M. Sami, Curr. Sci. 97, 887 (2009).

[2] R. Silva, J. S. Alcaniz and J. A. S. Lima, Int. J. Mod. Phys. D, 16, 469 (2007); R. Silva, R. S. Gonçalves, J. S. Alcaniz, and H. H. B. Silva, Astron. Astrop. 537, A11 (2012).

[3] J. A. S. Lima and J. S. Alcaniz, Phys. Lett. B 600, 191 (2004).

[4] P. F. Gonzalez-Diaz and C. L. Siguenza, Nucl. Phys. B 697, 363 (2004).

[5] J. A. S. Lima and S. H. Pereira, Phys. Rev. D 78, 083504 (2008).

[6] S. H. Pereira and J. A. S. Lima, Phys. Lett. B 669, 266 (2008).

[7] M. Chevallier and D. Polarski, Int. J. Mod. Phys. D 10, 213 (2001).

[8] E. V. Linder, Phys. Rev. Lett. 90, 091301 (2003).
[9] G. Efstathiou, Mon. Not. Roy. Astron. Soc. 310, 842 (1999).

[10] E. M. Barboza and J. S. Alcaniz, Phys. Lett. B 666, 415 (2008).

[11] V. B. Johri, astro-ph/0409161.

[12] D. Landau and E. M. Lifshitz, Fluid Mechanics (Pergamon Press, New York, 1959).

[13] S. Weinberg, Astrop. J. 168, 175 (1971).

[14] J. A. S. Lima and A. S. M. Germano, Phys. Lett. A 170, 373 (1992).

[15] R. Silva, J. A. S. Lima, and M. O. Calvão, Gen. Rel. Grav. 34, 865 (2002).

[16] Y. S. Myung, Phys. Lett. B 671, 216 (2009).

[17] H. B. Callen, Thermodynamics and an Introduction to Thermostatistics (2nd edition, Wiley, New York,1985).

[18] E. N. Saridakis, P. F. Gonzalez-Diaz, and C. L. Siguenza, Class. Quantum Grav. 26, 165003 (2009).

[19] G. Izquierdo and D. Pavon, Phys. Lett. B 633, 420 (2006).

[20] N. Bilic, Fortsch. Phys. 56, 363 (2008). 